\title{
ISSUE COVER
}




\title{
ANALYSIS OF IMPLICIT LES METHODS
}

\author{
ANDREW ASPDEN, NiKOS NiKIFORAKIS, \\ STUART DALZIEL AND JOHN B. BELL
}

\begin{abstract}
Implicit LES methods are numerical methods that capture the energy-containing and inertial ranges of turbulent flows, while relying on their own intrinsic dissipation to act as a subgrid model. We present a scheme-dependent Kolmogorov scaling analysis of the solutions produced by such methods. From this analysis we can define an effective Reynolds number for implicit LES simulations of inviscid flow. The approach can also be used to define an effective Reynolds number for under-resolved viscous simulations. Simulations of maintained homogeneous isotropic turbulence and the Taylor-Green vortex are presented to support this proposal and highlight similarities and differences with real-world viscous fluids. Direct comparison with data from high resolution DNS calculations provides validation of the effective viscosity and effective Kolmogorov length scale.
\end{abstract}

\section{Introduction}

The broad range of time and length scales present in high Reynolds number turbulent flows is prohibitively expensive for direct numerical simulation (DNS) to capture completely, and various techniques are used to attempt to overcome this problem. An approach that is receiving increasing attention is the use of a form of large eddy simulation (LES) known as implicit LES (ILES), where numerical schemes are used such that the inviscid energy cascade through the inertial range is captured accurately and the inherent numerical dissipation emulates the effects of the dynamics beyond the grid-scale cut-off. This approach was introduced in [5] (see also [6]), and referred to there as the Monotone Integrated Large Eddy Simulation (MILES), but has recently come to encompass a broader range of schemes under the name of ILES.

Many ILES codes are run without the inclusion of a viscous term, but others include a small amount of explicit viscosity (see, for example, [14]), with the viscous scales not fully resolved. Both approaches are considered to be ILES simulations in the literature, but although there is likely to be little difference for practical applications, there is a subtle difference in theory that will be discussed below.

MSC2000: 76F05, 76F65, 76M12, 76M55.

Keywords: Implicit LES, ILES, MILES. 
For this reason, we consider the three situations to be distinct: fully-resolved DNS, completely inviscid ILES, and ILES with viscosity (a subset of under-resolved DNS).

Since turbulence is characterised by high levels of fluctuating vorticity and, therefore, sharp velocity gradients similar to compressible shocks, the schemes used in ILES are inspired by ideas from shock-capturing schemes used for compressible flows. High-order, nonoscillatory, finite volume (NFV) schemes are particularly well suited here. In the early 90's, several authors published successful applications of these types of schemes, for example [29] and [36], but it was Boris [6] who first identified the convenient conspiracy, as it was later dubbed by Oran and Boris [27], specifically that the numerical dissipation inherent in these schemes acts at the small scales in a manner similar to a subgrid-scale model. Furthermore, the cell-averaging discretisation of the flow variables can be thought of as an implicit filter. Particular success has been found in free shear flows, where the influence of small-scale viscous dissipation is small; see, for example, [14]. Using a PPM-based method, Sytine et. al. [33] and Porter et. al. [30] showed that it is possible to recover energy spectra with a minus five-thirds decay. A collection of works that provides an overview of the technique, including a history and applications, can be found in [18].

Efforts to derive effective viscosities for ILES computations include Grinstein and Guirguis [17], who compared simulations using a flux-corrected transport algorithm with viscous theory for a two-dimensional (laminar) shear layer, and the modified equation analysis of Fureby and Grinstein [14], Grinstein and Fureby [16], Margolin and Rider [23; 24], who relate the modified equation to an implicit subgrid-scale model. The latter can lead to useful insights, but is heuristic in nature because modified equation analysis is valid at long wavelengths, but not at the length scales where the numerical dissipation is acting. An additional issue with modified equation analysis is that the analysis can become prohibitively cumbersome without drastic simplifying assumptions about the numerical method that may render the results meaningless. For example, the scheme used in the present study involves projections to enforce the incompressibility constraint [1], which cannot be readily incorporated into the analysis.

We will use the notion of a hypothetical fluid described by an ILES scheme and the term ILES fluid. The concept of a hypothetical fluid that arises from numerical simulation has been suggested before by Muschinski [26], where a similar framework is used. A Smagorinsky model is used there to specify an explicit eddy-viscosity coefficient, and so the analysis differs from the approach used here. What we do instead in this paper is use scaling to examine the turbulence that arises in an ILES fluid and compare it to real turbulence; we then use this comparison to validate the use of ILES in special cases and highlight its limitations. 


\section{Theory}

An incompressible homogeneous viscous flow is characterised by three parameters: the integral length scale $l$, the energy dissipation rate $\varepsilon$, and the fluid viscosity $\nu$. An important dimensionless parameter is the Reynolds number

$$
\operatorname{Re}_{\varepsilon} \equiv \frac{\varepsilon^{\frac{1}{3}} l^{\frac{4}{3}}}{v}
$$

Turbulence involves a cascade of kinetic energy from large scales (the ones that contain most of the energy) to small scales where the energy is dissipated by viscosity. Kolmogorov introduced the length scale

$$
\eta \equiv\left(\frac{v^{3}}{\varepsilon}\right)^{\frac{1}{4}}
$$

now known as the Kolmogorov length scale, which allows the Reynolds number to be written as

$$
\operatorname{Re}_{\varepsilon} \equiv\left(\frac{l}{\eta}\right)^{4 / 3},
$$

which is assumed here to be large.

In terms of a kinetic energy wavenumber spectrum, dimensional analysis suggests

$$
\frac{\kappa^{5 / 3} E}{\varepsilon^{2 / 3}}=\varphi_{\nu}(\kappa l, \kappa \eta),
$$

for some dimensionless function $\varphi_{\nu}$.

Kolmogorov's first similarity hypothesis states that at length scales $r \ll l$, that is, $\kappa l \gg 1$, the turbulent statistics are universal, determined uniquely by the energy dissipation rate and the viscosity, that is, $\kappa^{5 / 3} \varepsilon^{-2 / 3} E=\varphi(\kappa \eta)$. This range of scales is known as the universal equilibrium range. Furthermore, Kolmogorov's second similarity hypothesis states that for scales $\eta \ll r \ll l$, that is, $\kappa \eta \ll 1$, the statistics are independent of viscosity and so are determined uniquely by the energy dissipation rate, that is, there is complete similarity [3] and $\kappa^{5 / 3} \varepsilon^{-2 / 3} E=C_{\kappa}$, the Kolmogorov constant. This range of scales is known as the inertial (sub)range. The range of scales comparable to the Kolmogorov length scale, where kinetic energy is dissipated, is known as the dissipation (sub)range. This analysis implicitly assumes that the Reynolds number

$$
\operatorname{Re}_{\varepsilon}=\frac{(\kappa l)^{4 / 3}}{(\kappa \eta)^{4 / 3}}
$$

is large. 
Experimental observation and direct numerical simulation suggest a value for $C_{\kappa}$ between 1.2 and 2 . In the dissipation range, $\varphi_{v}$ has been observed to decay exponentially, for example in large-scale experiments by Saddoughi and Veeravalli [31] and in DNS calculations by Kerr [21].

The same approach can be taken for an ILES fluid. The characteristic parameters are now $\varepsilon$ and $l$ (as before), but now the computational cell width $\Delta x$ replaces the fluid viscosity. A new dimensionless group that can be formed with these parameters is $N \equiv l / \Delta x$, the number of cells across the integral length scale.

As before, dimensional analysis can be used to form an expression for the kinetic energy wavenumber spectrum:

$$
\frac{\kappa^{5 / 3} E}{\varepsilon^{2 / 3}}=\varphi_{i}(\kappa l, \kappa \Delta x),
$$

for some dimensionless function $\varphi_{i}$.

A theory can then be formed by analogy with Kolmogorov's theory. The first similarity hypothesis asserts that for length scales $r \ll l$, that is, $\kappa l \gg 1$, turbulent statistics are universal (for a particular numerical algorithm), determined solely by the energy dissipation rate and the computational cell width, that is, $\kappa^{5 / 3} \varepsilon^{-2 / 3} E=$ $\varphi_{i}(\kappa \Delta x)$; each numerical algorithm possesses a universal equilibrium range. The second similarity hypothesis can then be restated as, for scales $\Delta x \ll r \ll l$, that is, $\kappa \Delta x \ll 1$, the statistics are independent of the computational cell width, that is, again there is complete similarity and the normalised energy spectrum is a constant. Furthermore, assuming that the algorithm captures the advection term with sufficient accuracy, then this constant should be the same as in a viscous fluid, that is, the Kolmogorov constant.

There is no reason to assume that the dissipation range in an ILES fluid should be the same as in a viscous fluid, or that we can directly associate the transition between the inertial and dissipation subranges with $\Delta x$; at best, only the inertial range can be expected to be similar. It will be shown in the next section that normalising $\kappa$ with $\Delta x$ does not collapse the kinetic energy spectrum, and another length scale has to be used.

The extent of the inertial range is directly related to the Reynolds number, and so, given $l$, the shortest length scale in the inertial range will be indicative of the Reynolds number. Consider the integral

$$
\mathscr{D}=\frac{1}{V} \int_{V} \mathbf{u} \cdot \nabla^{2} \mathbf{u} \mathrm{d} V .
$$

In spectral space, $\mathbf{u} \cdot \nabla^{2} \mathbf{u}$ will resemble $\kappa^{2} E(\kappa)$, and so it can be expected to grow as $\kappa^{1 / 3}$ in the inertial range and decay exponentially in the dissipation range. Therefore, the dominant contribution to the integral $\mathscr{D}$ will come from length scales at the 
transition between the two subranges. Furthermore, in a viscous fluid, $\mathscr{D}$ arises in the kinetic energy equation, and is related to the energy dissipation rate according to $\varepsilon=\nu \mathscr{D}$, and so provides a link between an ILES fluid and a viscous fluid.

We propose that $D$ can be used to derive expressions for effective viscosity and effective Kolmogorov length scale that are loosely independent of the structure of the dissipation range, and are common to both an ILES fluid and a viscous fluid. Specifically, $v_{e}=\varepsilon / \mathscr{D}$ and $\eta_{e}=\varepsilon^{1 / 2} / \mathscr{D}^{3 / 4}$, which makes the relation $v_{e}^{3}=\varepsilon \eta_{e}^{4}$ consistent with a viscous fluid.

Dimensional analysis suggests that

$$
\frac{\eta_{e}}{\Delta x} \equiv \frac{\varepsilon^{1 / 2}}{\Delta x \mathscr{D}^{3 / 4}}=\Pi_{i}\left(\frac{l}{\Delta x}\right)
$$

for some dimensionless function $\Pi_{i}$, noting that $l / \Delta x \sim \operatorname{Re}_{e}^{3 / 4}$. Complete similarity would imply that $\eta_{e} / \Delta x$ is a constant, but this will be shown not to be the case, at least for the numerical scheme used here.

With this definition of an effective Kolmogorov length scale, the analogy with Kolmogorov's theory, Equation (6), can be recast in the form of Equation (4).

\section{Simulations}

The numerical code used throughout this study is IAMR, which is available from the Center for Computational Sciences and Engineering at the Lawrence Berkeley National Laboratory. IAMR is an incompressible, variable-density Navier-Stokes solver that is suitable for ILES calculations. Before briefly discussing the algorithmic approach in IAMR, we emphasise that our focus here is on developing a methodology for assessing ILES approaches, not on advocating for a particular method.

IAMR employs a finite volume approach with a two-step predictor-corrector method based on the unsplit second-order Godunov methodology introduced for gas dynamics by Colella [9]. The advective velocities are constructed using a monotonicity-limited fourth-order centred-difference slope approximation [8]. An intermediate MAC projection [4] is used to ensure these velocities are discretely divergence free before the flow variables are advected. Finally, an approximate projection [2] is used to enforce the divergence-free constraint on the updated velocity field. The overall algorithm is second-order in both space and time. (For further details, see [1] and the references therein.) It should be noted that secondorder accuracy is sufficient to be considered "high-order" and suitable for the ILES approach; see [11] or [19]. Moreover, Margolin et. al. [25] argue that second-order may be the only suitable way to construct an ILES scheme. 
The equations of motion are the standard incompressible Navier-Stokes equations,

$$
\begin{aligned}
\nabla \cdot \mathbf{u} & =0, \\
\frac{\partial \mathbf{u}}{\partial t}+(\mathbf{u} \cdot \nabla) \mathbf{u} & =-\frac{1}{\rho} \nabla p+v \nabla^{2} \mathbf{u}+\mathbf{F},
\end{aligned}
$$

where the density, velocity, pressure and viscosity are denoted by $\rho, \mathbf{u}, p$ and $v$, respectively, and $\mathbf{F}$ is a forcing term to be defined. In the following simulations, the viscosity is simply set to zero for inviscid ILES calculations, and the viscosity of under-resolved calculations will be specified when appropriate.

3.1. Maintained Homogeneous Isotopic Turbulence. Simulations of homogeneous isotropic turbulence were run in a triply-periodic cube ${ }^{1}$. To reduce the detrimental impact of long-range correlations that affect decaying turbulence (see [10], for example), a zero-mean time-dependent low-wavenumber forcing term was prescribed as

$$
\begin{array}{r}
\mathbf{F}(\mathbf{x}, t)=\sum_{|\kappa| \in[1,3]} \mathbf{a}_{i, j, k} \cos \left(f_{i, j, k} t+\psi_{i, j, k}\right) \cos \left(2 \pi \kappa_{i} x+p_{i, j, k}\right) \\
\times \cos \left(2 \pi \kappa_{j} y+q_{i, j, k}\right) \cos \left(2 \pi \kappa_{k} z+r_{i, j, k}\right),
\end{array}
$$

for random amplitudes $\mathbf{a}_{i, j, k} \in[0,1)$, frequencies $f_{i, j, k} \in[\pi, 2 \pi)$, and phases $\psi_{i, j, k}, p_{i, j, k}, q_{i, j, k}$ and $r_{i, j, k} \in[0,2 \pi)$. The flow was initiated with a low-level low-wavenumber velocity field, and unit density.

Inviscid (ILES) simulations were performed at resolutions from $32^{3}$ to $1024^{3}$. Viscous simulations were performed at $256^{3}$ (with viscosities of $v=10^{-2}, 10^{-3}$ and $10^{-4}$ ), $512^{3}$ (with viscosities of $10^{-3}$ and $10^{-4}$ ) and at $1024^{3}$ (with viscosities of $2.5 \times 10^{-4}$, and $10^{-4}$ ). Not all of the viscous simulations were expected to be fully-resolved; evaluating $\Pi_{v} \equiv \varepsilon^{1 / 2} /(\eta \mathscr{D})$ provides a way to establish which simulations are well-resolved $\left(\Pi_{v}=1\right)$ and which are not $\left(\Pi_{v}>1\right)$. Simulations were run until $t=8$, except for the $1024^{3}$ cases, which were run until times between 3 and 4 due to computational expense.

Figure 1(a-d) shows the evolution of the terms in the kinetic energy equation for all of the simulations; (a) is the total kinetic energy, (b) is the energy injected by the forcing term, (c) is the actual energy dissipation evaluated according to $\varepsilon=\phi-\mathrm{d} E / \mathrm{d} t$, where $\phi=(1 / V) \int \mathbf{u} \cdot \mathbf{F} \mathrm{d} V$, and (d) is $\mathscr{D}$ as defined in Equation (7). Inviscid and viscous runs are denoted by solid and broken lines, respectively, and colour denotes resolution. The flow passes through an initial transient as the energy cascade begins, the dissipation rate reaches a peak at around $t \approx 1$, and shortly

\footnotetext{
${ }^{1}$ Throughout this section, the units are arbitrary, and Reynolds numbers will be presented where appropriate.
} 

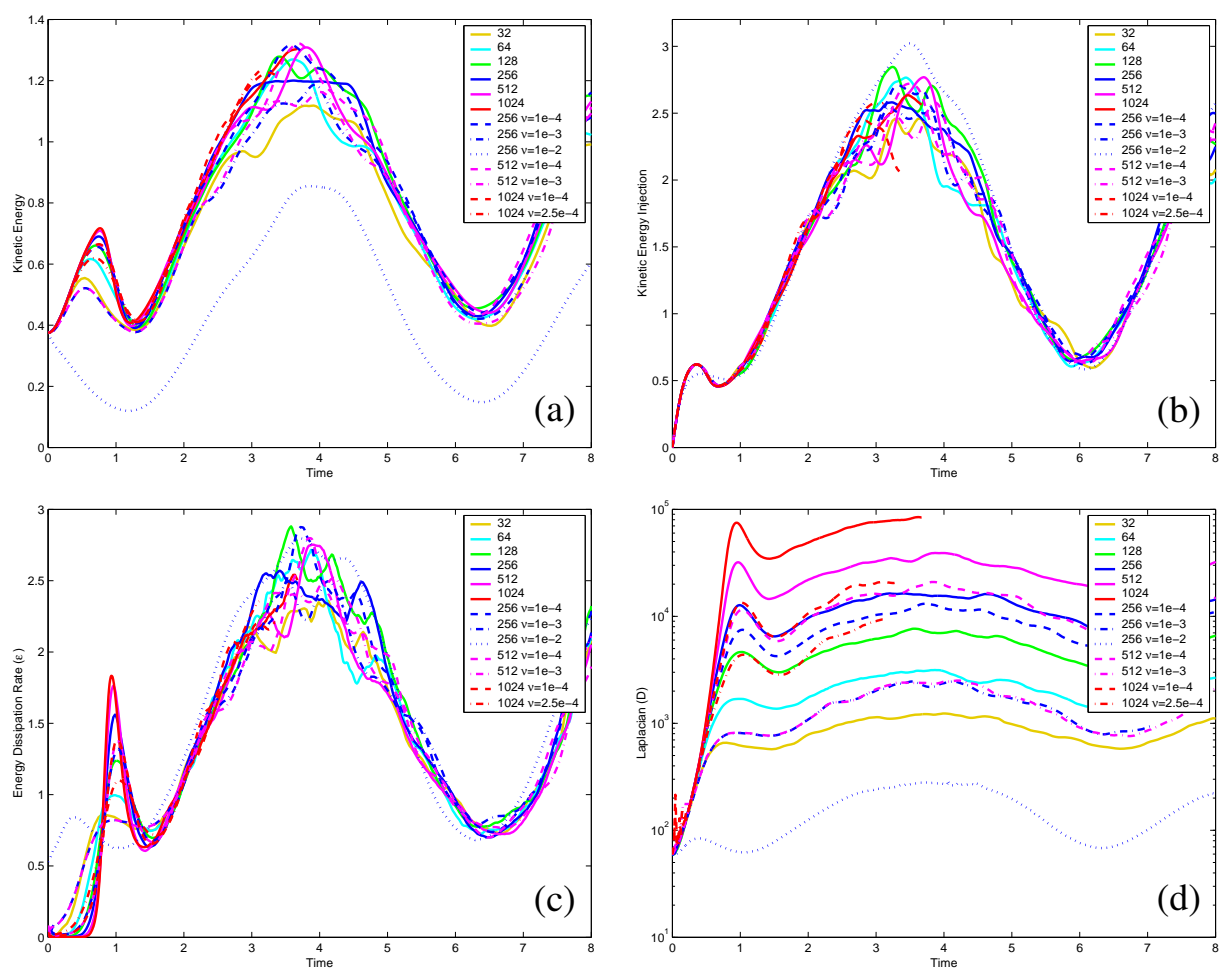

Figure 1. Evolution of (a) total kinetic energy $E$, (b) injection of kinetic energy due to the forcing term $\phi$, (c) energy dissipation rate $\varepsilon$, and (d) the Laplacian term $\mathscr{D}$. Inviscid and viscous simulations are denoted by solid and broken lines, respectively, and colour denotes resolution. The forcing term dominates the flow, but maintains a dissipation rate that is independent of resolution or viscosity.

thereafter becomes fully-developed. It is clear from these plots that the forcing term dominates the flow, but importantly maintains a time-dependent zero-mean velocity field with a dissipation rate that is independent of the resolution and viscosity. Figure 1 demonstrates that the resolution and viscosity affect only the small-scale energy dissipation; it is only the Laplacian term $\mathscr{D}$ in Figure 1(d) that is affected by changes in resolution or viscosity. In the most viscous case, the Reynolds number is too low for a sufficient separation of scales, and both the initial transient and the late-time evolution are heavily damped.

Figure 2(a) shows the evolution of the dimensionless quantity $\Pi_{i} \equiv \varepsilon^{1 / 2} /(\Delta x \mathscr{D})$ for the ILES simulations. For the resolutions presented here, the effective Kolmogorov length scale $\eta_{e}=\Pi_{i} \Delta x$ is between about one quarter and one third of 

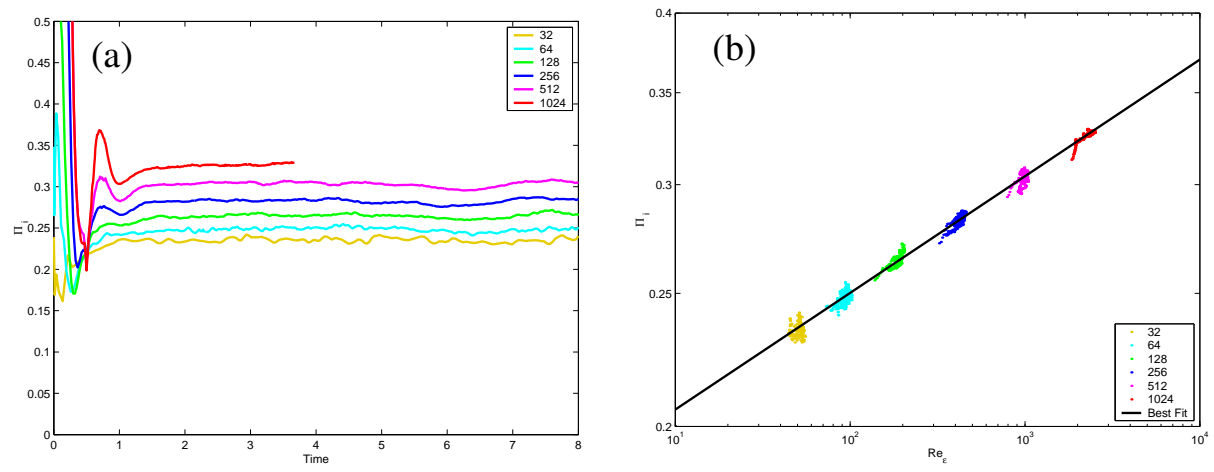

Figure 2. (a) Evolution of the dimensionless quantity $\Pi_{i}$ for the ILES simulations. In each case, the value settles to a constant after the initial transient. (b) Reynolds number dependence of $\Pi_{i}$. Data shown are for $t>1.2$. The best fit is $\Pi_{i}=0.169 \mathrm{Re}_{\varepsilon}^{0.085}$.

a computational cell width, which is much smaller than would be required for a well-resolved DNS calculation.

In each simulation, the value of $\Pi_{i}$ becomes approximately constant once the flow has become well-developed, but the lack of complete similarity alluded to in Section 2 is evident here. Figure 2(b) considers the dependence of $\Pi_{i}$ on the effective Reynolds number. The solid black line is a best fit to the power law $\Pi_{i}=0.169 \mathrm{Re}_{e}^{0.085}$, which can be stated equivalently in terms of resolution as $\Pi_{i}=0.203 N^{0.102}$, where $N$ is the number of cells across the integral length scale. This relationship demonstrates an incomplete similarity in Equation (8).

The source of this dependency is not clear, but two possible influences have been discounted: the numerical slope limiting used to preserve monotonicity, and the use of a large scale forcing term. The simulations were run without utilising slope limiting, but this only resulted in slightly smaller values for $\Pi_{i}$; the Reynolds number dependency remained. The decaying simulations in the next section will also be shown to possess a similar degree of dependency, discounting the forcing term.

It may be the case that the Reynolds number dependency is just a manifestation of an underlying limitation in relating an ILES simulation to a viscous fluid, which may be related to some other property of turbulence not considered here, such as intermittency. Recently, Sreenivasan [32] has argued that the resolution requirements for well-resolved DNS calculations grow at a rate that exceeds the three-quarters that natural scaling suggests, which may be related to the observations presented here. 


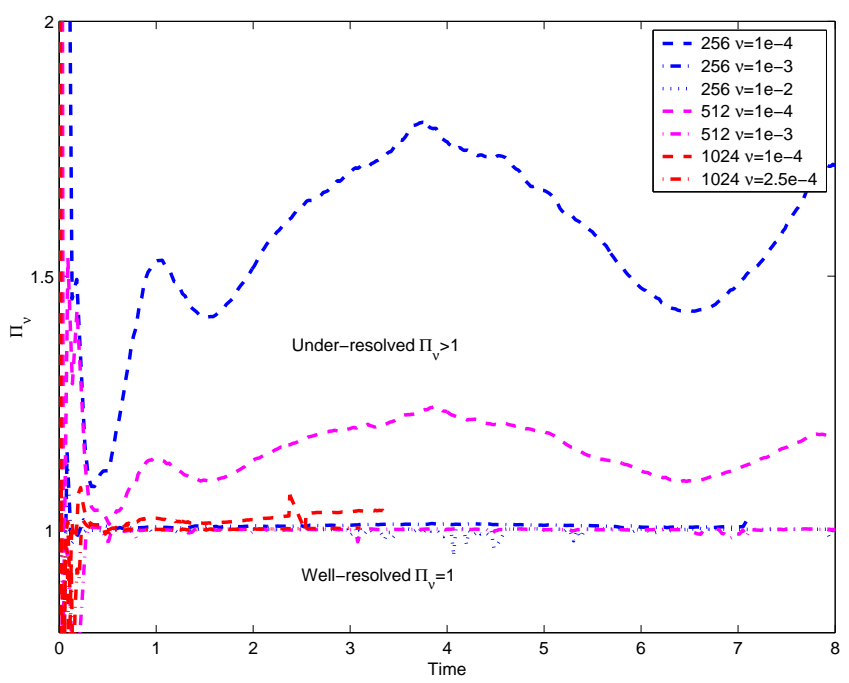

Figure 3. Evolution of the dimensionless quantity $\Pi_{v} . \Pi_{v} \approx 1$ corresponds to a well-resolved viscous calculation, and $\Pi_{v}>1$ denotes lack of resolution.

The measure $\Pi_{v}$, shown in Figure 3, distinguishes the viscous simulations that are well-resolved $\left(\Pi_{v} \approx 1\right)$ from those that are not $\left(\Pi_{v}>1\right)$. The simulations at $256^{3}$ with $v=10^{-2}, 512^{3}$ with $v=10^{-3}$ and $1024^{3}$ with $v=2.5 \times 10^{-4}$ are thought to be well-resolved as the maxima in $\Pi_{v}$ after the initial transient are 1.0052, 1.0045, and 1.0054, respectively (some error is expected due to evaluating the numerical derivatives for the temporal change in the total kinetic energy and the Laplacian). The simulations at $256^{3}$ with $v=10^{-3}$ and $1024^{3}$ with $v=10^{-4}$ are close, but not quite fully-resolved, where the maxima in $\Pi_{v}$ are 1.0145 and 1.0295 , respectively. The other two simulations at $v=10^{-4}$ are clearly not well-resolved.

Using the measured effective Kolmogorov length scales, the kinetic energy spectra can be normalised according to $\varepsilon^{-2 / 3} \eta_{e}^{-5 / 3} E\left(\kappa \eta_{e}\right)$, and are plotted in Figure 4; the low Reynolds number DNS simulation has been omitted. The same colour scheme as before has been used, and the dashed black line shows the theoretical inertial range decay $C_{\kappa} \kappa^{-5 / 3}$ with a Kolmogorov constant of $C_{\kappa}=2$. Even though ILES spectra are not expected to be identical to the viscous spectra, this normalisation appears to collapse both kinds of spectra in the universal equilibrium range. Not only do the ILES spectra collapse to a single profile, that profile does not appear to be too far removed from the viscous profile. In particular, it should be noted that the ILES spectra have a much shorter dissipation range than the viscous spectra; in the viscous simulations it is necessary to dedicate a significantly higher proportion of resolution to the dissipation range. Consequently, at the other end of the spectra, 


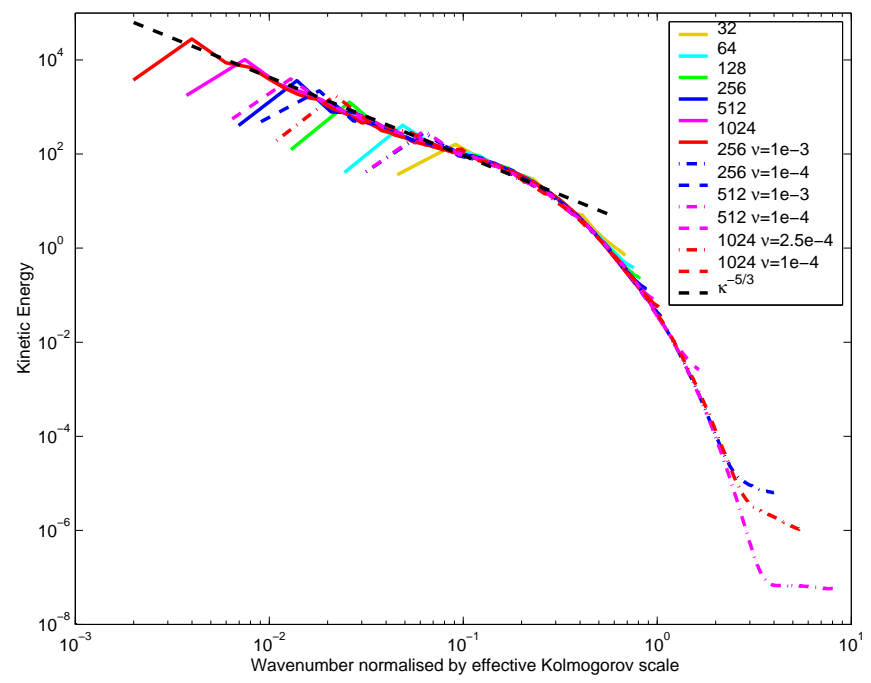

Figure 4. Normalised kinetic energy wavenumber spectra $\varepsilon^{-2 / 3} \eta^{-5 / 3} E(\kappa \eta)$. There is a clear collapse of both inviscid and viscous spectra. The high resolution simulation present an inertial range with decay close to the expected minus-five thirds. Note, in particular, how the viscous simulations are required to dedicate significantly more resolution to the dissipation range.

the ILES simulations have inertial ranges that extend to smaller wavenumbers than the viscous spectra, suggestive of higher Reynolds numbers. This behaviour can be seem more clearly in the compensated spectra, plotted in Figure 5(a,b). Here, another difference between the two types of spectra can be discerned: the dip around $\kappa \eta \approx 0.05$ appears to be slightly greater in the ILES case. Figure 5(c,d) plots the compensated spectra semilogarithmically to consider exponential decay in the dissipation range. The dashed black line is $A \exp (-\beta \kappa \eta)$, with $A=6.5$ and $\beta=5.2$, taken from the DNS simulations of Kerr [21] and the boundary-layer experiments of Saddoughi and Veeravalli [31], with which the viscous simulations are in very close agreement. The ILES simulations, however, present slightly different behaviour; there is a range with steeper decay followed by a flattening near the grid-scale. In summary, there are identifiable differences between the universal equilibrium ranges of ILES and viscous spectra, but these differences are not sufficient to disrupt the collapse presented in Figure 4, at least for the numerical scheme considered here. The scheme captures an inertial range close to the Kolmogorov constant, and the effective Kolmogorov length scale permits a normalisation that collapses the spectra to an equilibrium range that is universal for the scheme, and close to that of a real viscous fluid. 

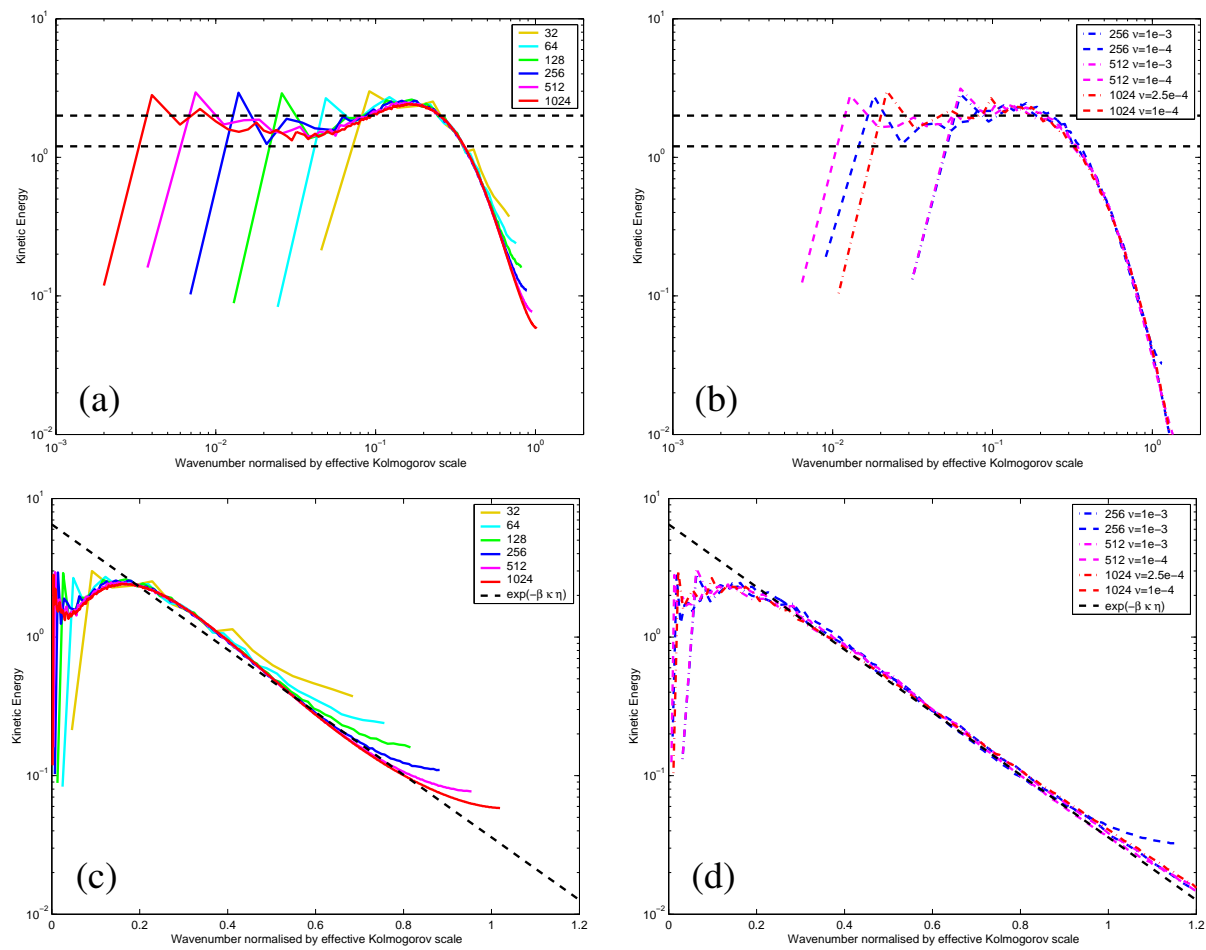

Figure 5. Compensated kinetic energy wavenumber spectra, normalised according to $\varepsilon^{-2 / 3} \kappa^{5 / 3} E(\kappa \eta)$. Inviscid spectra are shown in (a) and (c), and viscous spectra in (b) and (d). The inertial range is highlighted by logarithmic plots in (a) and (b) (the two black lines denote the range of values found in the literature for the Kolmogorov constant, that is, 1.2-2), and the dissipation range is highlighted by semilogarithmic plots in (c) and (d).

Figure 6(a) shows the evolution of the effective viscosity. The same measure is used in both the viscous and inviscid cases; the actual viscosity is not used explicitly, and so provides another measure of how well resolved the viscous calculations are. The vertical dashed line shows $t \approx 2.57$, which corresponds to the time at which the spectra are plotted in Figures 4-5. A key point to note here is that the extent to which the inertial range of each spectrum extends to low wavenumbers (Figure 4) corresponds directly to the effective viscosity at the time shown by the vertical dashed line (Figure 6a). As the Reynolds number increases, a larger inertial range is observed due to the greater separation of scales, so since all other quantities are approximately equal, the Reynolds number is represented by the effective viscosity, which follows exactly the same trend as the energy spectra at 

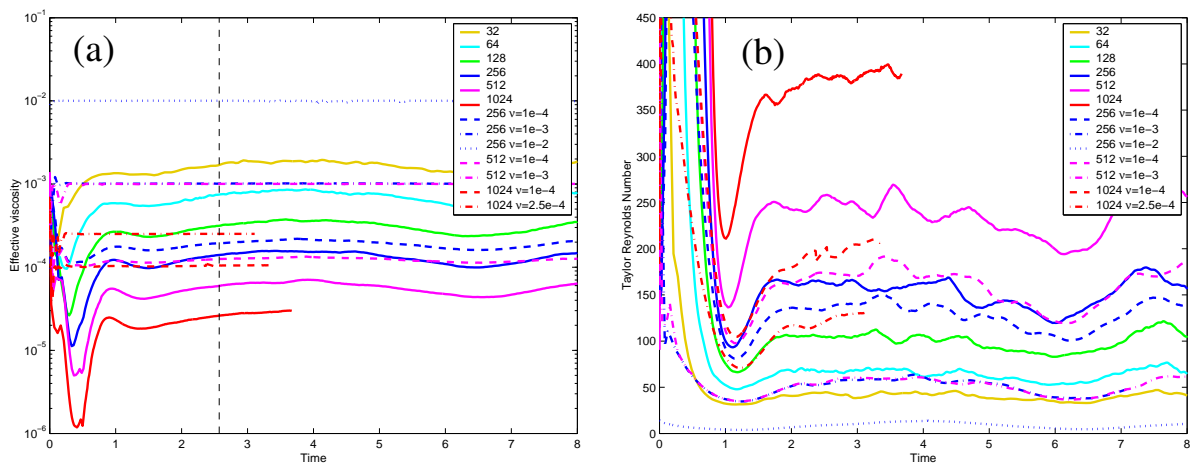

Figure 6. (a) Evolution of the effective viscosity $v_{e}$, for both the inviscid and viscous simulations; note the time-dependency of the ILES simulations, and that the effective viscosity of the underresolved viscous simulations does not agree with the specified viscosity. (b) Evolution of the Taylor Reynolds number.

large scales. This suggests that the effective viscosity that has been derived is an accurate representation of the flow; if an ILES simulation and a viscous fluid have inertial ranges that extend over the same range of wavenumbers, then the method outlined above provides a way of deriving the effective viscosity of the ILES fluid

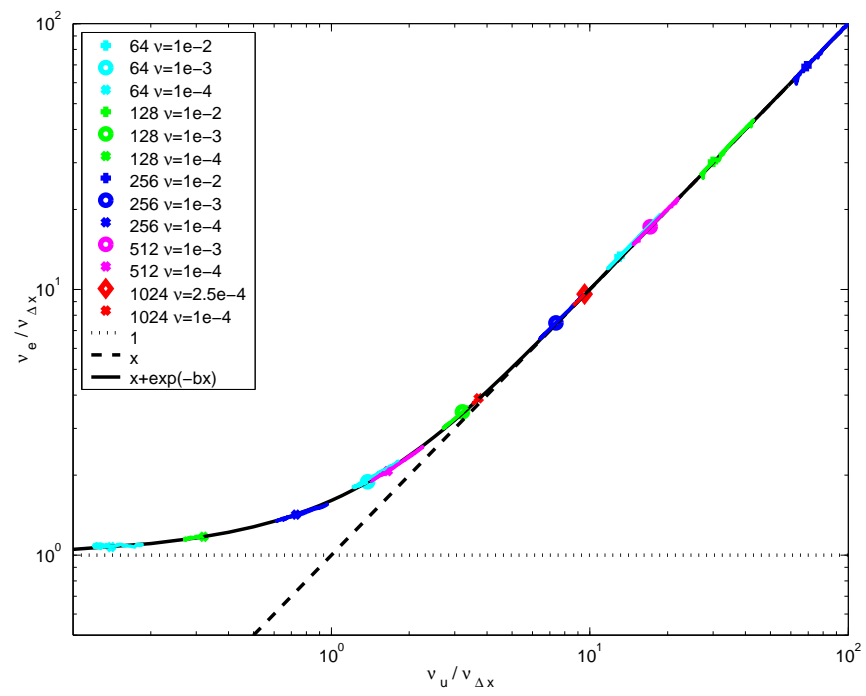

Figure 7. Examination of the dimensionless dependence of the effective viscosity on the specified viscosity in under-resolved viscous simulations, $v_{e} / v_{\Delta x}=f\left(v_{u} / v_{\Delta x}\right)$. 
corresponding to the true viscosity of the real fluid. The resulting effective Taylor Reynolds numbers

$$
\operatorname{Re}_{\lambda}=\frac{\hat{u} \lambda}{v_{e}} \quad \text { for } \quad \lambda^{2}=\frac{15 v_{e} \hat{u}^{2}}{\varepsilon}
$$

where $\hat{u}$ is the RMS velocity, are plotted in Figure 6(b).

Some ILES schemes are run with small amounts of viscosity, for example, Fureby and Grinstein [14]. Other viscous simulations are run using a nonoscillatory finite volume approach, where the resolution may not completely capture the dissipation range. To account for these circumstances, it is possible to extend the characterisation to the situation of a viscous ILES calculation. There are three measures of viscosity that need to be considered: the specified (under-resolved) viscosity $v_{u}$, the effective viscosity for an inviscid simulation at that resolution $v_{\Delta x}$, and the resulting effective viscosity $v_{e}$. Dimensional considerations suggest a functional dependence of the form

$$
\frac{v_{e}}{v_{\Delta x}}=f\left(\frac{v_{u}}{v_{\Delta x}}\right)
$$

for some dimensionless function $f$, where $f(x) \rightarrow 1$ as $x \rightarrow 0$, and $f(x) \rightarrow x$ as $x \rightarrow \infty$.

To investigate this dependence, a variety of under-resolved simulations were run in addition to those already presented, the results of which are shown in Figure 7. For each simulation, the marker denotes the time $t=2.57$, and the surrounding points shows time dependence. The dotted line shows $f(x)=1$ (the inviscid limit), the dashed line is $f(x)=x$ (the well-resolved viscous limit), and the solid black line is the heuristic candidate function

$$
f(x)=x+\exp (-b x)
$$

which naturally satisfies the restrictions on (9). The value of $b$ shown here is $1 / 2$. There is clear agreement for all simulations, which suggests that an a priori prediction for the effective viscosity of an under-resolved viscous calculation using this scheme can be written as

$$
v_{e}=v_{u}+v_{\Delta x} \exp \left(-\frac{1}{2} \frac{v_{u}}{v_{\Delta x}}\right)
$$

3.2. The Taylor-Green Vortex. The Taylor-Green vortex [34] has become a popular test case for ILES methods (see the recent studies of Drikakis et. al. [12] and Hickel et. al. [20]) and so has been investigated here. Following [12], the domain used was a triply-periodic cube of length $2 \pi$. The velocity field was initialised 

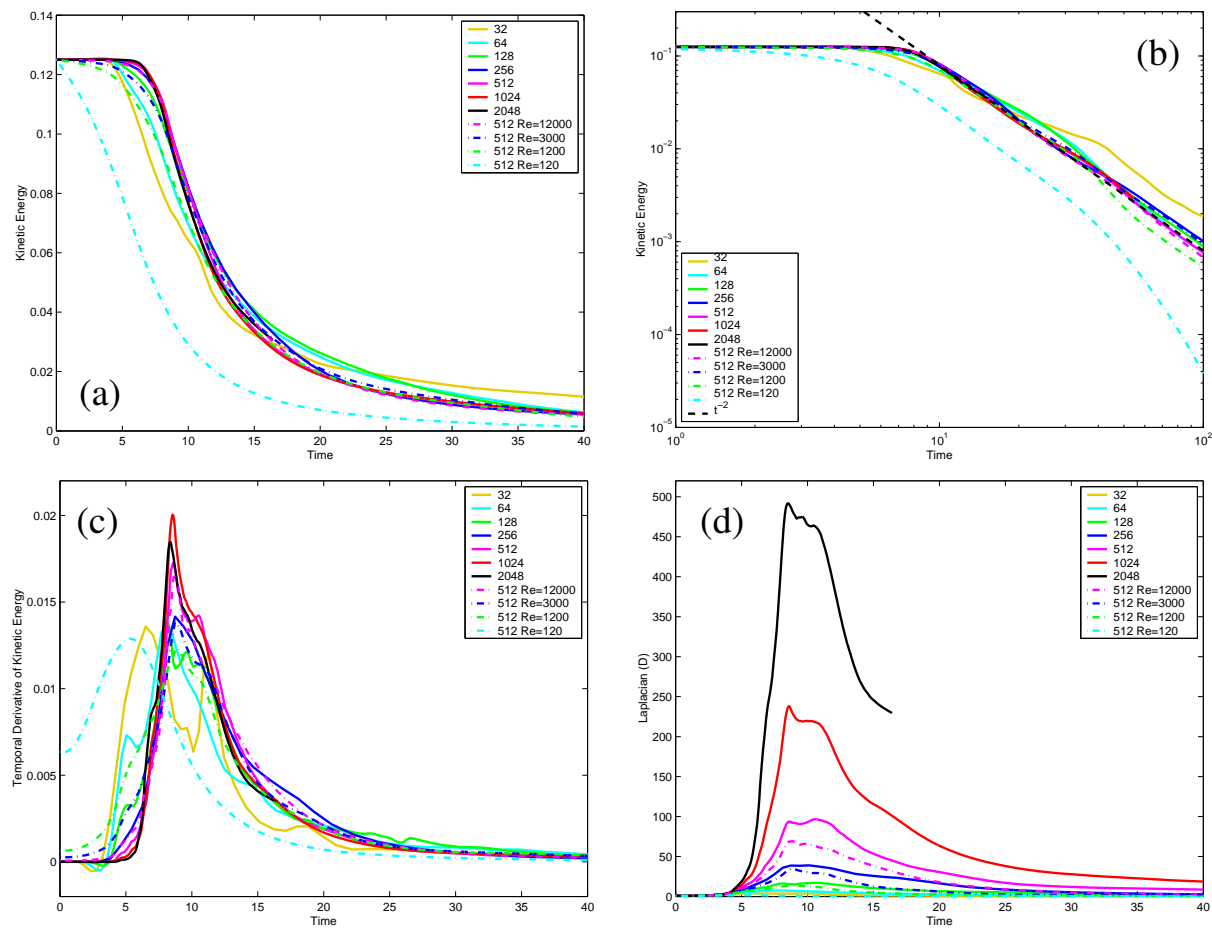

Figure 8. Evolution of the terms in the kinetic energy equation for the Taylor-Green vortex: $(a, b)$ total kinetic energy, $(c)$ temporal change in kinetic energy, (d) Laplacian term $\mathscr{D}$. The solid lines are the inviscid calculations and the dash-dotted lines are the viscous calculations.

according to

$$
\mathbf{u}_{0}(\mathbf{x})=u_{0}\left(\begin{array}{c}
\cos (k x) \sin (k y) \cos (k z) \\
-\sin (k x) \cos (k y) \cos (k z) \\
0
\end{array}\right),
$$

where $k=1$. The inherent symmetry of the problem can be exploited to reduce the domain size by a factor of 8 . Simulations were run at effective resolutions of $32^{3}$ to $2048^{3}$. Viscous simulations were run at an effective resolution of $512^{3}$, at Reynolds numbers of approximately 120, 1200, 3000, and 12000, where the Reynolds number is defined to be $\operatorname{Re}=u_{0} / k v$, corresponding to the initial conditions; as in the previous section, this range of Reynolds numbers spans the range from being too viscous for a separation of scales to being under-resolved at the given resolution. Throughout this section velocities will be nondimensionalised by $u_{0}$ and lengths by $k^{-1}$. 

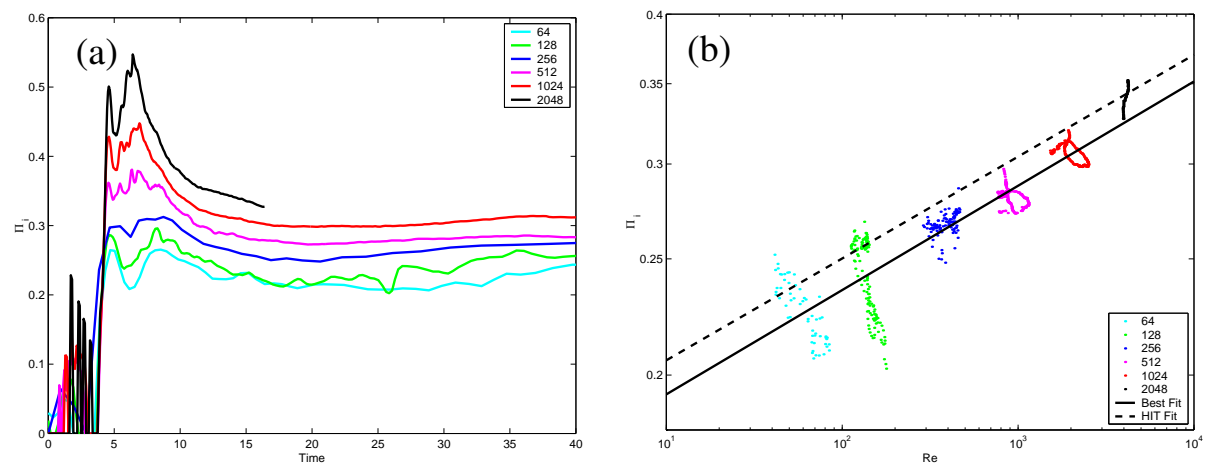

Figure 9. (a) Evolution of the dimensionless parameters $\Pi_{i}$. (b) Dependence of $\Pi_{i}$ on $\operatorname{Re}_{\varepsilon}$. Data shown are for $t>12$. Solid black line denotes best fit, $\Pi_{i}=0.152 \mathrm{Re}_{\varepsilon}^{0.087}$. Dashed black line is the best fit from the previous section.

Figures $8(a, b)$ show the evolution of the normalised total kinetic energy; (c) shows the temporal derivative of the kinetic energy, and (d) shows the Laplacian term D. At early times, kinetic energy is conserved (in the inviscid and high Reynolds number cases), then as the cascade process begins, a growth in the Laplacian term is observed along with a corresponding decay in kinetic energy. The energy dissipation rate reaches a peak at around dimensionless time $t \approx 9$, and the late-time energy decay follows $t^{-2}$, characteristic of the Taylor-Green vortex. The most viscous case prevents transition to turbulence, and the vortex spins down at a rate that can be seen to be faster than $t^{-2}$ at late times.

In the ILES results of Drikakis et. al. [12] and the DNS results of Brachet [7] at a Reynolds number of 5000, the energy dissipation is observed to peak at a value around 0.016, and it is suggested in [13], for example, that a limit independent of Reynolds number is being approached. However, the peak energy dissipation in the $1024^{3}$ case presented in Figure 8(c) is approximately $25 \%$ higher, and so suggests that much higher Reynolds numbers will be needed to draw any definitive conclusions. The simulation at $2048^{3}$ does not attain a peak as high as the $1024^{3}$ case. This is because the Taylor-Green vortex is extremely sensitive to shear instabilities, which are damped at lower resolutions.

Figure 9(a) shows the dimensionless quantity $\Pi_{i}$. There is a slightly greater variability in the value of $\Pi_{i}$ for each simulation than there was for the simulations in the previous section, and again there is a dependence on resolution. Figure 9(b) shows the Reynolds number dependency of $\Pi_{i}$ for each simulation. The solid black line denotes the best fit to the data, which is of the form $\Pi_{i}=0.152 \operatorname{Re}_{\varepsilon}^{0.087}$. The dashed black line shows the best fit from the simulations from the previous section, 

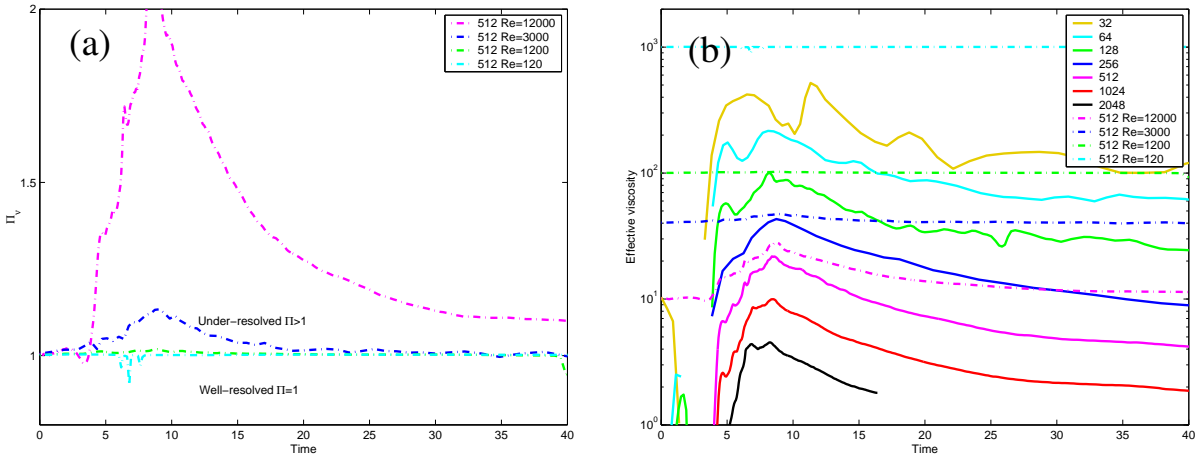

Figure 10. (a) Evolution of the dimensionless parameter $\Pi_{v}$. (b) Evolution of the effective viscosity $v_{e}$.

which has a similar power-law growth, but a slightly higher coefficient (recall the best fit from the previous section was $\Pi_{i}=0.169 \mathrm{Re}_{\varepsilon}^{0.085}$ ).

Figure 10 shows the dimensionless quantity $\Pi_{v}$ (a), and the effective viscosities (b). The least viscous case is clearly identified as being under-resolved, but because the flow is decaying, the simulation approaches the well-resolved limit at late times. The $\operatorname{Re}=3000$ case appears to be marginally under-resolved, but the other two viscous cases appear to be well-resolved.

Figure 11 shows the kinetic energy spectra, normalised as before using the effective Kolmogorov length scale and energy dissipation rate, along with reference spectra from the maintained homogeneous isotropic turbulence simulations from the previous section, specifically the inviscid simulation at $1024^{3}$ and the well-resolved viscous simulation at $512^{3}$ with $v=10^{-3}$; the dashed black line denotes $C_{\kappa} \kappa^{-5 / 3}$, with $C_{\kappa}=2$. Again, the normalisation collapses the data well, not only the viscous and inviscid simulations, but also the maintained and decaying flows; a universal equilibrium range is indeed recovered in these ILES simulations, and appears to be similar to that recovered in a viscous fluid.

Compensated spectra are shown in Figure $12(a, b)$ for two different times: (a) is at $t \approx 16.4$, the latest time that the highest resolution case was run, and (b) is at $t \approx 50$. At the first time, the compensated spectra demonstrate that the decay is indeed close to minus five-thirds and the data compares well with the maintained spectra. However, at the later time, the decay is less than five-thirds. Note, in particular, that even well-resolved DNS spectra do not achieve a minus five-thirds decay. This is a consequence, and one of the drawbacks, of decaying turbulence; it should be emphasised that it is not a consequence of using the ILES approach. Without a source of energy at the large scales, a much larger domain and separation of scales is required for truly free decay; Pope [28] suggests that a lower bound on 


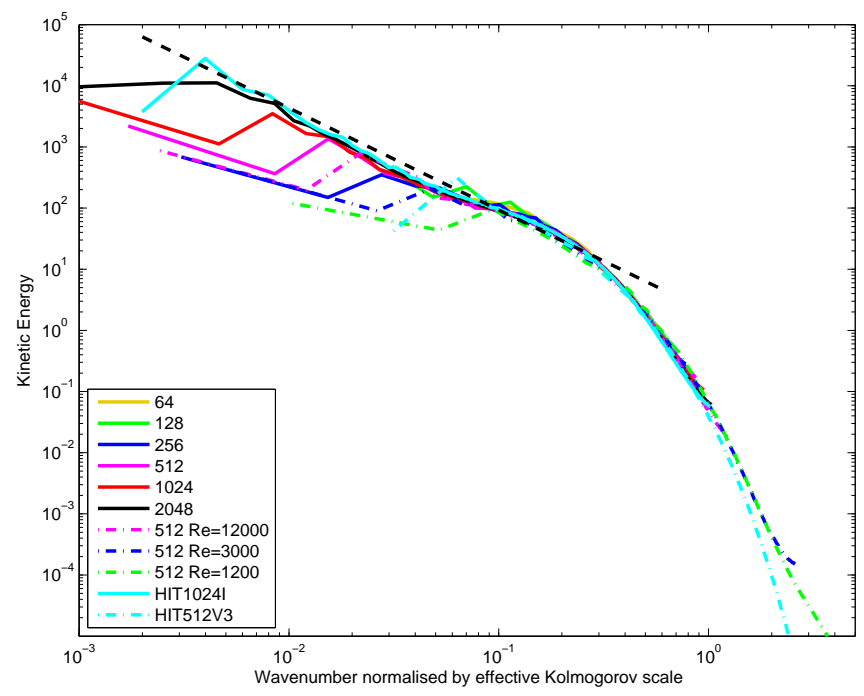

Figure 11. Kinetic energy wavenumber spectra, $\varepsilon^{-2 / 3} \eta^{-5 / 3} E(\kappa \eta)$, at dimensionless time $t \approx 16.4$. Two maintained simulations from the previous section (labelled HIT) are shown for comparison; specifically the inviscid simulation at $1024^{3}$ and the viscous simulation at $512^{3}$ with $v=10^{-3}$.

the domain size is around eight integral length scales, and Davidson [10] argues that the factor should be more like twenty to forty; refer, in particular, to the section on "the dangers of periodicity" in [10]. This is the likely cause of the lack of universality in $\Pi_{i}$ observed here. It also highlights a difference between ILES and
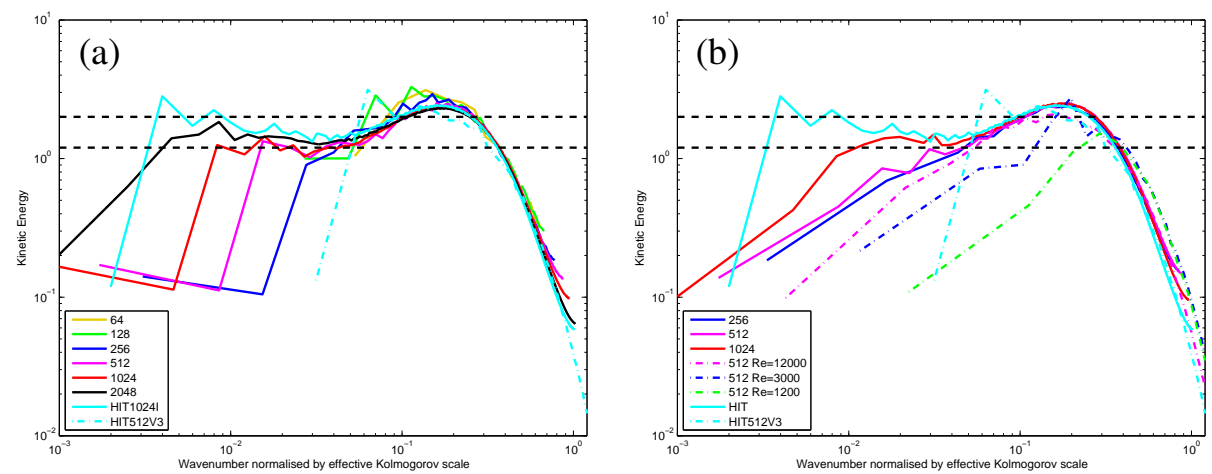

Figure 12. Compensated kinetic energy wavenumber spectra for the Taylor-Green vortex, normalised according to $\varepsilon^{-2 / 3} \kappa^{5 / 3} E(\kappa \eta)$. (a) $t \approx 16.4$, (b) $t \approx 50$. 



Figure 13. Vertical slices showing magnitude of vorticity in a inviscid calculation at $256^{3}$ (top) and in a viscous calculation at $512^{3}$ with $\mathrm{Re}=3000$ (bottom) at three different times $t \approx 10$ (left), $t \approx 100$ (middle), $t \approx 1000$ (right). Note that the small-scale structure is removed by viscosity in the finite Reynolds number case, but persists in the ILES calculation due to the imposed separation of scales.

traditional LES, where the aim it is to extend the minus-five thirds decay to, or as close as possible to, the cut-off length scale, and may explain the behaviour observed by Garnier [15].

Another key difference between ILES simulations and real-world viscous or DNS fluids arises in flows that are decaying, and is due to the fixed effective Kolmogorov length scale in the ILES case. In a viscous fluid, as the flow decays, the energy dissipation rate drops, and so the Kolmogorov length scale increases according to Equation (2). This cannot happen in an ILES fluid; instead, the effective viscosity decreases. The consequence of this is that all small-scale structure is removed from the viscous simulation, but high wavenumber velocity gradients persist in the ILES case. This is highlighted in Figure 13, which shows vertical slices of the magnitude of vorticity at $t=10,100$ and 1000 for both a viscous calculation $\left(512^{3}\right.$, $\operatorname{Re} \approx 3000$ ) and an inviscid calculation $\left(256^{3}\right)$. Naturally, the plots are similar at the early time, but at later times diffusive effects dominate the viscous flow and 


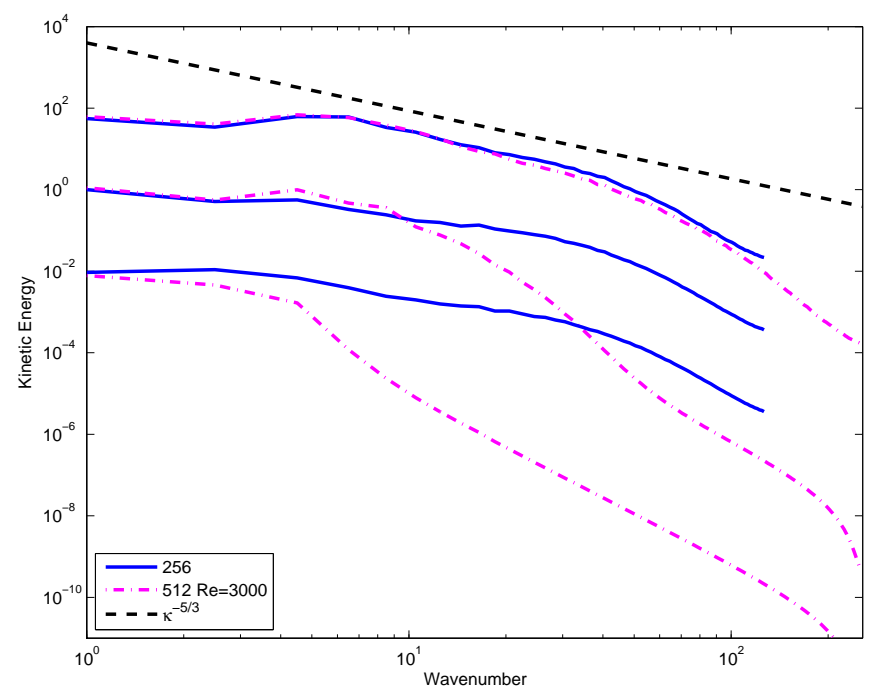

Figure 14. Unnormalised kinetic energy spectra corresponding to the vorticity slices shown in Figure 13 illustrating differences in relaminarisation for different approaches. The three pairs of spectra show how energy decreases at the three different times. Note in particular the lack of energy at large wavenumbers for the viscous case.

the Kolmogorov length scale grows. The ILES plots are qualitatively similar at the intermediate and late times because of the fixed effective Kolmogorov length scale. Unnormalised kinetic energy spectra are shown in Figure 14. Again, at the early time, the agreement is close, but it is clear that at later times the distribution of energy across the scales is significantly different.

This is the reason we make the distinction between inviscid ILES simulations and ILES simulations with viscosity. If an ILES simulation is performed including a small amount of explicit viscosity, then in a decaying flow, that viscosity will eventually begin to play a role. If the viscosity is kept constant the small-scale structure will eventually be removed, regardless of how small the viscosity is.

\section{Discussion and conclusions}

In this paper, we have presented a scaling analysis of implicit LES methods in general, and proposed a methodology for characterising individual ILES schemes. This was achieved by drawing an analogy with the description of viscous fluids given by Kolmogorov [22]. More specifically, an ILES method can be characterised in terms of an equilibrium range that is universal to that approach, and is determined uniquely by the energy dissipation rate $\varepsilon$, as in a viscous fluid, and the computational cell 
width $\Delta x$, which replaces the fluid viscosity as the characteristic measure of smallscale energy dissipation. By using dimensional analysis, a single dimensionless parameter $\Pi_{i} \equiv \varepsilon^{1 / 2} /(\Delta x \mathscr{D})$ was derived that provides a characteristic estimate of the effect of the numerical dissipation on the small scales in a developed turbulent flow for an ILES scheme. To mimic the relationships of viscous fluids, an effective Kolmogorov length scale and effective viscosity can be written as $\eta_{e}=\Pi_{i} \Delta x$ and $v_{e}=\varepsilon^{1 / 3} \Pi_{i}{ }^{4 / 3} \Delta x^{4 / 3}$.

This approach differs philosophically from previous work as it uses a posteriori diagnostics to evaluate the characteristic estimate $\Pi_{i}$, complementing the previous a priori approaches that consider modified equation analysis. The present methodology has the benefit that it assesses a scheme's performance based on the results, not on heuristic predictions, and the framework can be applied easily to any ILES scheme however complicated the algorithm.

For the ILES scheme presented here, in the maintained homogeneous turbulence simulations, it was found that $\Pi_{i}=0.169 \mathrm{Re}_{\varepsilon}^{0.085}$ or equivalently $\Pi_{i}=0.203 N^{0.102}$, where $N$ is the number of computational cells across the integral length scale, which means that the effective Kolmogorov length scales were between approximately one fifth and one third of a computational cell width. The values obtained for $\Pi_{i}$ were slightly smaller for the decaying Taylor-Green vortex. This is likely due to a lack of independence from the large scales. For this reason, we believe that maintained turbulence is a more suitable test case for characterising an ILES scheme, and is more likely to give results consistent with more realistic applications.

The effective Kolmogorov length scale and effective viscosity for an ILES simulation were used to normalise kinetic energy spectra, and it was demonstrated, by comparison with well-resolved DNS calculations, that an ILES flow with an effective viscosity close to that of a DNS calculation had an inertial range that spanned the same range of wavenumbers; this measure of effective viscosity is an accurate representation of an ILES fluid.

Furthermore, under-resolved simulations were investigated, and it was found that a simple expression could be formulated to predict the effective viscosity a priori:

$$
v_{e}=v_{u}+v_{\Delta x} \exp \left(-\frac{1}{2} \frac{v_{u}}{v_{\Delta x}}\right)
$$

This demonstrates that the variation between fully-resolved DNS and completely inviscid ILES simulations is continuous, except for a distinction made for completely inviscid simulations for late-time decaying flows, summarised below. The expression can be used to specify the effective viscosity in marginally resolved DNS studies using an NFV scheme. 
Differences were observed between ILES and viscous spectra, both at the high wavenumber end of the inertial range, and within the dissipation range. These differences were small, and only observed under close scrutiny, the physical implications of which are not yet understood.

A key difference is that the effective viscosity depends on the energy dissipation rate, which is local in both time and space, and so knowledge of some kind of measure of the dissipation rate (in whatever average sense applies to the flow) is required. This means that not only can the effective viscosity vary in time, it can also be different in different regions of the flow. In a turbulent jet, for example, a higher effective viscosity will be observed along the jet axis than at the jet edge because of the decreasing dissipation rate with radius; see Townsend [35] for example. Furthermore, the Reynolds number will increase with streamwise distance, rather than remain constant as expected in a round jet.

In decaying flows, a significant consequence is that a completely inviscid ILES flow cannot undergo relaminarisation; there is no final period of decay. In a decaying viscous flow, as the energy dissipation rate drops, the Kolmogorov length scale increases as small-scale structure is removed by viscosity. This cannot happen in an inviscid ILES fluid; there is an imposed separation of scales due to the fixed Kolmogorov length scale. The vorticity field will decay, but cannot become smooth; small-scale structure will persist for all time. However, for ILES schemes run with a small amount of explicit viscosity, a final period of decay will eventually be observed, regardless of how small the viscosity is.

An expression for the effective viscosity can be written in the form $v_{e}=\varepsilon / \mathscr{D}$, which has no a priori reason to hold true in an ILES flow. It may be the case that because this scheme is second-order accurate (Margolin et. al. [25] argue that second-order may be the only suitable way to construct an ILES scheme), then $\mathscr{D}$ is indeed a close measure of how energy is removed from the system. But the question remains whether this will be the case in other ILES codes.

Regardless, it can be argued that $\mathscr{D}$ provides a suitable way to measure energy dissipation (even if nonunique) because the wavenumber spectrum of the integrand will be similar to $\kappa^{2} E(\kappa)$. If an ILES scheme captures the inviscid energy cascade, then $E(\kappa) \sim \kappa^{-5 / 3}$ in the inertial range, and at some wavenumber, energy is dissipated and decreases rapidly with wavenumber. Therefore, the spectrum of the integrand of $\mathscr{D}$ will peak at a length scale around the transition between the integral and dissipation ranges. Thus the dominant contribution to the integral will come from around this length scale, and will not be dominated by the details of the scheme-dependent dissipation scales, just their physical location. This length scale is also strongly related to the Reynolds number and given the exact relation for viscous fluids, we expect $\mathscr{D}$ to provide the link between numerical dissipation and effective viscosity for any ILES algorithm. 
The parameter $\Pi_{i}$ is the dimensionally correct scaling to characterise a scheme, and the expression is independent of the numerical scheme. It specifically captures (through $\mathscr{D}$ ) the effect of the numerical scheme on small scale dissipation. Its value (and any Reynolds number dependence) will vary between different algorithmic approaches. For example, we speculate that higher-order schemes will present spectra with a longer inertial range, i.e. the dissipation range will be shorter and begin closer the grid-scale, and consequently present smaller values for $\Pi_{i}$. It may also be the case that in the compensated spectrum, the peak energy at the bottleneck between the inertial and dissipation ranges will be greater than was found here and the dip in the spectra observed at smaller wavenumbers will also be exaggerated.

An issue that has not been addressed here is how the parameter $\Pi_{i}$ will behave for inhomogeneous flows. Consider a jet or shear layer, where there is a transition from fully-developed turbulent flow to laminar quiescence. Both the numerator $\varepsilon$ and the denominator $\mathscr{D}$ tend to zero. We intend to consider this transition in future work.

\section{Acknowledgements}

AJA's time at DAMTP was funded by an AWE/EPSRC CASE award, and at LBNL by a Seaborg Fellowship. JBB was supported by the Office of Advanced Scientific Computing Research, Applied Mathematics Research Program of the U.S. Department of Energy under Contract No. DE-AC02-05CH11231. Calculations have been performed on Franklin at NERSC, Atlas at LLNL, Jaguar at NCCS, Blue Oak at AWE and Franklin at the Cambridge-Cranfield HPCF. AJA would also like to thank David Youngs, Robin Williams, Colm Caulfield and Malcolm Andrews for their useful discussions.

\section{References}

[1] A. S. Almgren, J. B. Bell, P. Colella, L. H. Howell, and M. L. Welcome, A conservative adaptive projection method for the variable density incompressible Navier-Stokes equations, Journal of Computational Physics 142 (1998), no. 1, 1-46.

[2] A. S. Almgren, J. B. Bell, and W. Y. Crutchfield, Approximate projection methods: Part I. inviscid analysis, SIAM J. Sci. Comput. 22 (2000).

[3] G. I. Barenblatt, Scaling, self-similarity, and intermediate asymptotics, Cambridge University Press, 1996.

[4] J. B. Bell, P. Colella, and L. H. Howell, An efficient second-order projection method for viscous incompressible flow, 10th AIAA Computational Fluid Dynamics Conference, Honolulu, 1991.

[5] J. P. Boris, On large eddy simulation using subgrid turbulence models comment 1, Whither Turbulence? Turbulence at the Crossroads (J. L. Lumley, ed.), Lecture Notes in Physics, Berlin Springer Verlag, vol. 357, 1990, pp. 344-353.

[6] J. P. Boris, F. F. Grinstein, E. S. Oran, and R. L. Kolbe, New insights into large eddy simulation, Fluid Dynamics Research 10 (1992), 199-229. 
[7] M. E. Brachet, Direct simulation of three-dimensional turbulence in the Taylor-Green vortex, Fluid Dynamics Research 8 (1992), 1-8.

[8] P. Colella, A direct Eulerian MUSCL scheme for gas dynamics, SIAM J. Sci. Stat. Comput. 6 (1985), 104-117.

[9] _ A multidimensional second order godunov scheme for conservation laws, J. Comput. Phys. 87 (1990), 171-200.

[10] P. A. Davidson, Turbulence : an introduction for scientists and engineers, Oxford University Press, 2004.

[11] D. Drikakis, Advances in turbulent flow computations using high-resolution methods, Progress in Aerospace Sciences 39 (2003), 405-424.

[12] D. Drikakis, C. Fureby, F. F. Grinstein, and D. L. Youngs, Simulation of transition and turbulence decay in the Taylor-Green vortex, Journal of Turbulence 8 (2007), 1-12.

[13] U. Frisch, Turbulence. The legacy of A.N. Kolmogorov, Cambridge University Press, 1995.

[14] C. Fureby and F. F. Grinstein, Monotonically integrated large eddy simulation of free shear flows, AIAA Journal 37 (1999), 544-556.

[15] E. Garnier, M. Mossi, P. Sagaut, P. Comte, and M. Deville, On the use of shock-capturing schemes for large-eddy simulation, Journal of Computational Physics 153 (1999), 273-311.

[16] F. F. Grinstein and C. Fureby, Recent progress on MILES for high reynolds number flows, Journal of Fluids Engineering 124 (2002), 848-861.

[17] F. F. Grinstein and R. H. Guirguis, Effective viscosity in the simulation of spatially evolving shear flows with monotonic FCT models, Journal of Computational Physics 101 (1992), 165-175.

[18] F. F. Grinstein, L. G. Margolin, and W. J. Rider, Implicit large eddy simulation, Cambridge University Press, 2007.

[19] A. Harten, High resolution schemes for hyperbolic conservation laws, Journal of Computational Physics 49 (1983), 357-93.

[20] S. Hickel, N. A. Adams, and J. A. Domaradzki, An adaptive local deconvolution method for implicit LES, Journal of Computational Physics 213 (2006), 413-436.

[21] R. M. Kerr, Velocity, scalar and transfer spectra in numerial turbulence, Journal of Fluid Mechanics 211 (1990), 309-332.

[22] A. N. Kolmogorov, (a) The local structure of turbulence in incompressible viscous fluid for very large Reynolds numbers, and $(b)$ dissipation of energy in the locally isotropic turbulence, Royal Society of London Proceedings Series A 434 (1991), (a) 9-13, and (b) 15-17.

[23] L. G. Margolin and W. J. Rider, A rationale for implicit turbulence modelling, International Journal for Numerical Methods in Fluids 39 (2002), 821-841.

[24] _ The design and construction of implicit LES methods, International Journal for Numerical Methods in Fluids 47 (2004), 1173-1179.

[25] L. G. Margolin, W. J. Rider, and F. F. Grinstein, Modeling turbulent flow with implicit LES, Journal of Turbulence 7 (2006), 1-27.

[26] A. Muschinski, A similarity theory of locally homogeneous and isotropic turbulence generated by a smagorinsky-type les, Journal of Fluid Mechanics 325 (1996), 239-260.

[27] E. S. Oran and J. P. Boris, Computing turbulent shear flows - a convenient conspiracy, Computers in Physics 7 (1993), 523.

[28] S. B. Pope, Turbulent flows, Cambridge University Press, September 2000. 
[29] D. H. Porter, A. Pouquet, and P. R. Woodward, Three-dimensional supersonic homogeneous turbulence: A numerical study, Phys. Rev. Lett. 68 (1992), 3156.

[30] Kolmogorov-like spectra in decaying three-dimensional supersonic flows, Physics of Fluids 6 (1994), 2133-2142.

[31] S. G. Saddoughi and S. V. Veeravalli, Local isotropy in turbulent boundary layers at high reynolds number, Journal of Fluid Mechanics 268 (1994), 333-372.

[32] K. R. Sreenivasan, Resolution effects in direct numerical simulations of turbulence, Turbulent Mixing and Beyond Workshop, Trieste, Italy, July 2007.

[33] I. V. Sytine, D. H. Porter, P. R. Woodward, S. W. Hodson, and K.-H. Winkler, Convergence tests for the piecewise parabolic method and Navier-Stokes solutions for homogeneous compressible turbulence, Journal of Computational Physics 158 (2000), 225-238.

[34] G. I. Taylor and A. E. Green, Mechanism of the production of small eddies from large ones, Proceedings of the Royal Society of London, Series A 158 (1937), 499-521.

[35] A. A. Townsend, The structure of turbulent shear flow, Cambridge University Press, 1967.

[36] D. L. Youngs, Three-dimensional numerical simulation fo turbulent mixing by Rayleigh-Taylor instability, Physics of Fluids A 4 (1991), 1312-20.

Received July 28, 2008.

ANDREW ASPDEN: AJAspden@lbl.gov

Lawrence Berkeley National Laboratory, 1 Cyclotron Road, MS 50A-1148, Berkeley, CA 94720, United States

and

Department of Applied Mathematics and Theoretical Physics, University of Cambridge, Cambridge CB3 OWA, United Kingdom

NIKOS NIKIFORAKIS: nn10005@cam.ac.uk

Department of Applied Mathematics and Theoretical Physics, University of Cambridge, Cambridge CB3 OWA, United Kingdom

STUART DALZIEL: s.dalziel@damtp.cam.ac.uk

Department of Applied Mathematics and Theoretical Physics, University of Cambridge, Cambridge CB3 OWA, United Kingdom

JOHN B. BELL: jbbell@lbl.gov

Lawrence Berkeley National Laboratory, 1 Cyclotron Road, MS 50A-1148, Berkeley, CA 94720, United States 